\title{
Pregnancy and multiple sclerosis
}

\author{
J A FRITH, J G McLEOD \\ From the Department of Medicine, University of Sydney, N.S.W., Australia
}

SUMMARY The effect of pregnancy on the relapse rate of multiple sclerosis has been investigated. Obstetric and relapse histories of 52 women with clinically definite multiple sclerosis were obtained; there were 101 pregnancies of which 85 were at risk of a multiple sclerosis relapse. By comparing the number of relapses in the pregnancy period ( 9 months of pregnancy and 6 months immediately post partum) with the number in non-pregnancy periods for the same women, no increased risk of relapse during the pregnancy period was demonstrated. However, of the relapses that did occur during the pregnancy period, there was a significant reduction in the number of relapses in the first and second trimesters.

The propensity for the onset of multiple sclerosis to occur in the reproductive years and for the disease to affect a greater proportion of females implies that many people who have multiple sclerosis will also have pregnancies. Consequently questions arise from the concerned patient regarding the effect of pregnancy on disease course and the effect of the disease on the course and outcome of pregnancy. The present study addresses the effect pregnancy has on the course of multiple sclerosis and looks specifically at the effect of pregnancy on relapse rate.

\section{Methods}

\section{Patients}

A population of 431 female patients with clinically definite multiple sclerosis according to the Schumacher criteria ${ }^{1}$ was studied. These women resided in New South Wales and were taking part in a therapeutic trial of transfer factor (TF) which involved regular clinical review. A questionnaire with explanatory letter was sent to each patient to identify those who had become pregnant since 1980 .

If the patient had had a pregnancy since 1980 , she was interviewed by telephone; the interview format had previously been piloted on 12 mothers without multiple sclerosis. From the interview, information on each patient was obtained regarding her obstetric history as well as history of multiple sclerosis onset, disease course and relapses. This information was confirmed by data from medical records compiled as part of a national epidemiology survey of multiple sclerosis in Australia. The design of this study was approved by the Royal Prince Alfred Hospital Medical Ethics Review Committee.

Address for reprint requests: Dr J G McLeod, Department of Medicine, University of Sydney, N.S.W. 2006, Australia.

Received 25 August 1987 and in revised form 17 November 1987. Accepted 24 November 1987

\section{Statistical Analysis}

For the purposes of statistical analysis, a relapse of multiple sclerosis was defined as a worsening of symptoms or signs or the occurrence of new symptoms or signs that lasted more than 24 hours. ${ }^{1}$ The number of relapses of multiple sclerosis for a patient was defined as the total number of attacks excluding the initial episode. The pregnancy period was the 9 months of pregnancy (divided into three trimesters) and two 3 month periods immediately post partum. More than one relapse could occur in any one year, year of onset or pregnancy period.

The relapse rate was defined as the number of relapses divided by the number of years of observation. The significance of the difference in number of relapses between pregnancy and non-pregnancy periods was compared by Chi-square test and the Mantel-Haenszel test stratified on individual patients. When relapses were subdivided according to maternal age of onset and duration of disease in a way similar to that used by Millar and colleagues ${ }^{2}$ significance was assessed by Chi-square analysis with Yates correction factor applied where appropriate. The significance of the distribution of relapses within the pregnancy period was assessed by Chi-square analysis and the application of $95 \%$ confidence intervals. For all statistical analyses the level of significance was taken as $\mathrm{p}<0.05$.

\section{Results}

Of the 431 patients who received the questionnaire, 407 replied, giving a $94 \%$ response rate. Fifty-two women had become pregnant since 1980 and were interviewed by telephone. These 52 women had 15 spontaneous abortions, 15 terminations and 101 pregnancies of greater than 20 weeks' gestation. Therefore there were 2.52 pregnancies per woman. Four of the pregnancies of greater than 20 weeks' gestation ended in perinatal death which led to a rate of live births $/ 1,000$ women of 1,865 . This was com- 
parable to the total fertility rate of 1,931 live births/1,000 women in New South Wales calculated on data for $1983 .{ }^{3}$

Of these 52 women with multiple sclerosis, relapse data were not available on two women who had in total three pregnancies of greater than 20 weeks' gestation.

The onset of multiple sclerosis occurred during pregnancy in four patients in the 1st trimester, 3rd trimester, 1 st 3 months post partum and 2 nd 3 months post partum respectively.

Forty relapses occurred during 85 pregnancy periods (106.25 years) giving a relapse rate of $0 \cdot 38 /$ year (table 1). There were 193 relapses that occurred outside the pregnancy periods for these same women. The total time of follow up, not including pregnancy periods, was 362.75 years which gave a relapse rate for non-pregnancy periods of 0.53 /year. The difference between the number of relapses in the pregnancy and non-pregnancy periods was significant with Chi-square analysis $(p<0.05)$ but did not reach significance with the application of the MantelHaenszel test stratified on individual patients $(\mathrm{p}<0 \cdot 1)$.

Analysis of relapse data was repeated taking into account the woman's age at onset and her duration of disease (table 2). In no subgroup did the number of observed relapses in the pregnancy period exceed the number of expected relapses. No significant difference in the number of relapses was found when pregnancy and non-pregnancy periods for each subgroup were compared.

The number of relapses in each trimester is shown in table 3. This distribution of relapses within the

Table 1 Relapse data

\begin{tabular}{llll}
\hline & $\begin{array}{l}\text { Number of } \\
\text { Relapses }\end{array}$ & $\begin{array}{l}\text { Years of } \\
\text { Observation }\end{array}$ & $\begin{array}{l}\text { Relapse } \\
\text { Rate/year }\end{array}$ \\
\hline $\begin{array}{l}\text { Pregnancy period } \\
\begin{array}{l}\text { Non-pregnancy } \\
\text { period }\end{array}\end{array}$ & $40^{*}$ & 106.25 & 0.38 \\
\hline
\end{tabular}

*The number of relapses in pregnancy and non-pregnancy periods was compared with the Chi-square test $(4.012, p<0.05)$ and the Mantel-Haenszel test stratified on individual patients $(3.025$, $\mathrm{p}<0 \cdot 1)$. pregnancy period was significantly non uniform (Chisquare test $\mathrm{p}<0.01$ ). Only $15 \%$ of the relapses occurred in the first 6 months of pregnancy. The $95 \%$

Table 3 Distribution of relapses during the pregnancy period

\begin{tabular}{ccl}
\hline Period & $\begin{array}{l}\text { Number of Relapses } \\
\text { Observed }\end{array}$ & $\begin{array}{l}\text { Number of Relapses } \\
\text { Expected }\end{array}$ \\
\hline Trimester 1 & 3 & 8 \\
2 & 3 & 8 \\
3 & 13 & 8 \\
Post Partum & & \\
Ist 3 months & 14 & 8 \\
2nd 3 months & 7 & 8
\end{tabular}

The expected number of relapses for each trimester and post partum period was the 40 relapses that occurred in the pregnancy period divided equally between each of the five subdivisions of the pregnancy period.

The distribution of relapses was not homogeneous (Chi-square test $p<0.01$, degrees of freedom $=4$ )

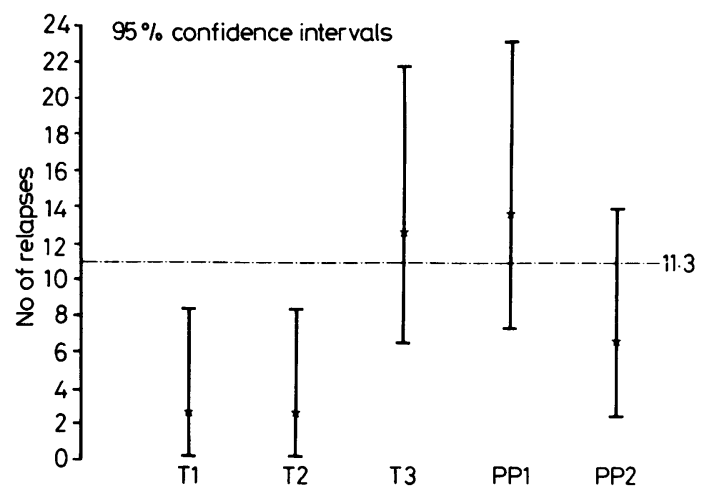

Fig Observed number of relapses in each trimester or postpartum period (*) with $95 \%$ confidence intervals (vertical bars) compared with the expected number of relapses (horizontal dotted line) for each trimester and postpartum period. The expected value (11.3) was derived from the relapse rate per year for the same women when not pregnant. With a non-pregnancy relapse rate of $0 \cdot 53 /$ year, in the $106 \cdot 25$ years of pregnancy, $56 \cdot 3$ relapses were expected to occur giving 11.3 relapses expected in each three months of the pregnancy period.

$T 1=1$ st trimester $T 2=2 n d$ trimester $T 3=3 r d$ trimester $P P 1=1$ st 3 months post partum $P P 2=2$ nd 3 months post partum

Table 2 Number of relapses (observed and expected) when considering age at onset and duration of disease

\begin{tabular}{|c|c|c|c|c|c|}
\hline \multicolumn{2}{|c|}{$\begin{array}{l}\text { Age at Onset } \\
\text { Duration of Disease }\end{array}$} & $\begin{array}{l}<25 y r \\
0-5\end{array}$ & $\underset{6^{+}}{<25 y r}$ & ${ }_{0-5}^{25^{+}} \mathrm{yr}$ & $25^{+} y r$ \\
\hline $\begin{array}{l}\text { Pregnancy } \\
\text { Non-pregnancy }\end{array}$ & $\begin{array}{l}\mathrm{O} \\
\mathrm{E} \\
\mathrm{O} \\
\mathrm{E}\end{array}$ & $\begin{array}{l}2 \\
4 \cdot 125 \\
9 \\
6 \cdot 875\end{array}$ & $\begin{array}{l}29 \\
36 \cdot 78 \\
145 \\
137 \cdot 22\end{array}$ & $\begin{array}{l}1 \\
1 \cdot 625 \\
12 \\
11 \cdot 375\end{array}$ & $\begin{array}{l}8 \\
9 \cdot 67 \\
27 \\
25 \cdot 33\end{array}$ \\
\hline
\end{tabular}

$\mathrm{O}=$ Observed $\mathrm{E}=$ Expected

Relapse rate/year was derived by dividing the total number of pregnancy and non-pregnancy relapses for each subgroup by the total years of follow up for that subgroup. The expected number of relapses was obtained by multiplying relapse rate/year by the number of years of follow up in each of the pregnancy and non-pregnancy periods for the subgroup. 
confidence intervals for the number of relapses in each trimester and post partum period are displayed in the fig and compared with that expected from the nonpregnancy rate. There were significantly fewer relapses in the 1st and 2nd trimesters; the remainder of the pregnancy period gave similar rates of relapse to the non-pregnancy period.

\section{Discussion}

In this investigation of the effect of pregnancy on the relapse rate of multiple sclerosis there was a $94 \%$ response rate for both the questionnaire and telephone interview. The 52 female multiple sclerosis patients interviewed had 101 pregnancies of greater than 20 weeks' gestation giving a fertility rate similar to that of the New South Wales population. Analysis of relapse data for these women showed that there was no increased risk of relapse during the pregnancy period. However, those relapses that did occur in the pregnancy period tended to cluster in the 3 rd trimester and first 3 months post partum with a significant reduction in number of relapses in the first two trimesters of pregnancy.

A review of the literature revealed that until 1950 most articles on the subject of pregnancy and multiple sclerosis appeared in German journals and reported an adverse effect of pregnancy on the disease (reviewed by Tillman ${ }^{4}$ ); some articles concluded by recommending "interruption of pregnancy" to prevent disease deterioration.

In 1950 Tillman $^{4}$ performed a retrospective review of patient records concerning 52 women who had 70 pregnancies. He concluded that pregnancy was not responsible for the onset of multiple sclerosis and that the exacerbations which occurred in pregnancy or the post partum were not necessarily "a cause and effect relationship". McAlpine and Compston ${ }^{5}$ reviewed 33 pregnancies in 24 patients and concluded that the relapse rate in the pregnancy periods did not differ significantly from the general relapse rate. These conclusions were supported by the work of Sweeney ${ }^{6}$ published in 1953.

In 1959, Millar and colleagues ${ }^{2}$ performed a retrospective review. Dealing with 45 pregnancy-associated relapses in 170 at-risk pregnancies they came to the conclusion that there was an increased relapse rate in the pregnancy period but reduced relapse rate in the non-pregnancy period leading to no overall change in frequency of relapse. Schapira and colleagues ${ }^{7}$ found an increased relapse rate in pregnancy years. Both groups of workers ${ }^{2}$ noted that the relapse rate was increased particularly in the post partum period.

There was a subsequent paucity of published literature until Ghezzi and Caputo ${ }^{8}$ in 1981 and Poser and Poser $^{9}$ in 1983 demonstrated no overall adverse effect of multiple sclerosis in pregnancy but again they did show an increased relapse rate post partum.

More recently, Korn-Lubetzki and colleagues ${ }^{10}$ studied the number of relapses occurring in each trimester of pregnancy as well as the number occurring in the post partum period. They examined 85 relapses in 199 pregnancies experienced by 66 Israeli women and found a "drastic reduction of the relapse rate in the 3rd trimester $(p=0.001)$ as well as an increase post partum $(p<0.001)$ ". However, overall there was no increase in the number of relapses in the pregnancy period for these Israeli women when compared with the relapse rate experienced by a control group.

All studies thus far have been retrospective in design and consequently most authors have compared the relapse rates of patients in pregnancy periods to the relapse rates of different groups of women acting as controls. Although the Australian study was also retrospective the data were sufficiently well documented to enable the relapse rate of women during their pregnancy periods to be compared with the relapse rate for the same women when they were not pregnant.

In conclusion, the Australian study of pregnancy and non-pregnancy periods in 52 women acting as their own controls, demonstrated that there was no increased risk of relapse of multiple sclerosis in the pregnancy period. This is in agreement with the data from Korn-Lubetzki's group. ${ }^{10}$ However, there was a significant reduction in the number of relapses experienced by Australian women in the first two trimesters of pregnancy while for Israeli women the number of relapses was significantly reduced in the 3rd trimester of pregnancy and increased in the first 3 months post partum.

In the space of 40 years, the current view of the effect of pregnancy on multiple sclerosis has changed from one of a deleterious influence possibly necessitating therapeutic abortion to a point where this present study, along with that of Korn-Lubetzki, ${ }^{10}$ has demonstrated that there is no increased risk of relapse in pregnancy and in fact there may be a beneficial effect on the disease at some stages of pregnancy.

These clinical data, coupled with experimental evidence that pregnancy in guinea pigs and rats exerts a suppressive influence on the development of experimental allergic encephalomyelitis, ${ }^{11}$ indicates that it may be important to examine more closely the effect of pregnancy on multiple sclerosis. Hormonal and/or immunological changes of the mother in pregnancy could be of relevance to proposed theories of aetiology and pathogenesis of multiple sclerosis.

We thank Drs Paul Lancaster and Susan Quine of the National Perinatal Statistics Unit for advice regarding 
formulation of the telephone interview. Thanks are also due to Dr Simon Hammond, Department of Medicine, University of Sydney for allowing access to the National Multiple Sclerosis Epidemiology Survey patient records and Professor Geoffrey Berry, School of Public Health and Tropical Medicine, for providing statistical advice. Dr Frith is an NH\&MRC Postgraduate Medical Research Scholar.

\section{References}

1 Schumacher G, Beebe G, Kibler R, et al. Problems of experimental trials of therapy in multiple sclerosis: report by the panel on the evaluation of experimental trials of therapy in multiple sclerosis. Ann NY Acad Sci 1965;122:552-68.

2 Millar J, Allison R, Cheeseman E, Merrett J. Pregnancy as a factor influencing relapse in disseminated sclerosis. Brain 1959;82:417-26.

3 Australian Bureau of Statistics. Births, New South Wales,
1985. Sydney: ABS, 1986. Catalogue no. 3306.1.

4 Tillman A. The effect of pregnancy on multiple sclerosis and its management. Res Publ Assoc Res Nerv Ment Dis 1950;28:548-82.

5 McAlpine D, Compston N. Some aspects of the natural history of disseminated sclerosis. $Q \mathrm{~J}$ Med 1952;21:135-67.

6 Sweeney W. Pregnancy and multiple sclerosis. Am J Obstet Gynecol 1953;66:124-30.

7 Schapira K, Poskanzer D, Newell D, Miller H. Marriage, pregnancy and multiple sclerosis. Brain 1966;89: 419-28.

8 Ghezzi A, Caputo D. Pregnancy: a factor influencing the course of multiple sclerosis? Eur Neurol 1981;20:115-7.

9 Poser S, Poser W. Multiple sclerosis and gestation. Neurology 1983;33:1422-7.

10 Korn-Lubetzki I, Kahana E, Cooper G, Abramsky O. Activity of multiple sclerosis during pregnancy and puerperium. Ann Neurol 1984;16:229-31.

11 Keith A. Effect of pregnancy on experimental allergic encephalo-myelitis in guinea pigs and rats. $J$ Neurol $S c i$ 1978;38:317-26. 